\title{
STABILITY ANALYSIS OF THE MAIN SPINDLE VIBRATIONS IN TRANSVERSAL DIRECTION AT CNC LATHE
}

\author{
Dr. Eng. POPESCU DANIEL ${ }^{*}$ 四
}

${ }^{1}$ University of Craiova, Faculty of Mechanics, Romania

DOI: https://doi.org/10.29121/IJOEST.v4.i3.2020.80

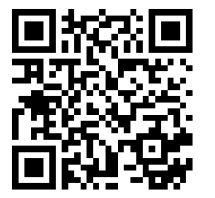

Article Type: Research Article

Article Citation: Dr. Eng. Popescu Daniel. (2020). STABILITY

ANALYSIS OF THE MAIN SPINDLE VIBRATIONS IN TRANSVERSAL DIRECTION AT CNC LATHE. International Journal of Engineering Science Technologies, 4(3), 30-34. https://doi.org/10.29121/IJOEST.v 4.i3.2020.80

Received Date: 24 April 2020

Accepted Date: 29 May 2020

Keywords:

Stability Analysis

Vibration

Main Spindle

CNC Lathe

Mihailov Criterion

\begin{abstract}
The paper presents an original method for stability analysis of the main spindle variations in transversal direction at CNC lathe, which by means of the Mihailov criterion and adequate software packages, establishes the dynamic instability character due to the specific processing conditions and to the grinding regime parameters. This creates the premises for rational design of the turning process, as well as for the structural design of the turning tool.
\end{abstract}

\section{INTRODUCTION}

Increased demands on the quality of finished industrial products due to increasing market competition has led to design and usage of high productivity modern machines and equipment like CNC machines, processing centres but also integrated systems of CNC machines such as flexible processing cells and flexible processing systems [1], [2], [3].

For this reason, studying the vibrations and analyzing ways of reducing them plays an important role in designing high performance equipment [4].

Ensuring a low level of vibrations creates the premises for achieving a sufficient stability degree for high precision processing, with superior surface quality and increased productivity [5], [6].

All these eventually add up into complex products and parts with a high degree of automation and mechanization. 


\section{ESTABLISHING THE METHOD FOR ANALYZING STABILITY OF THE MAIN SPINDLE IN CASE OF TRANSVERSAL VIBRATIONS AL CNC LATHE}

For this purpose, it is necessary to determine the equations that describe the vibration movement of the main spindle at CNC lathe. These are in fact a system of $1^{\text {st }}$ degree differential equations.

For analysis of the vibrations in transversal direction we take into account the solutions of the system of first order differential equations that generate the vibration movement of the main spindle [7]:

$$
\begin{aligned}
& \bar{v}_{3}^{\mathrm{i}}=\mathrm{e}_{11} \mathrm{a}_{1}^{\mathrm{i}}+\mathrm{e}_{12} \mathrm{a}_{2}^{\mathrm{i}}+\mathrm{e}_{13} \mathrm{a}_{3}^{\mathrm{i}}+\mathrm{e}_{14} \mathrm{a}_{4}^{\mathrm{i}} \\
& \overline{\mathrm{v}}_{3,1}^{\mathrm{i}}=\mathrm{e}_{21} \mathrm{a}_{1}^{\mathrm{i}}+\mathrm{e}_{22} \mathrm{a}_{2}^{\mathrm{i}}+\mathrm{e}_{23} \mathrm{a}_{3}^{\mathrm{i}}+\mathrm{e}_{24} \mathrm{a}_{4}^{\mathrm{i}} \\
& \overline{\mathrm{v}}_{3,11}^{\mathrm{i}}=\mathrm{e}_{31} \mathrm{a}_{1}^{\mathrm{i}}+\mathrm{e}_{32} \mathrm{a}_{2}^{\mathrm{i}}+\mathrm{e}_{33} \mathrm{a}_{3}^{\mathrm{i}}+\mathrm{e}_{34} \mathrm{a}_{4}^{\mathrm{i}} \\
& \overline{\mathrm{v}}_{3,111}^{\mathrm{i}}=\mathrm{e}_{41} \mathrm{a}_{1}^{\mathrm{i}}+\mathrm{e}_{42} \mathrm{a}_{2}^{\mathrm{i}}+\mathrm{e}_{43} \mathrm{a}_{3}^{\mathrm{i}}+\mathrm{e}_{44} \mathrm{a}_{4}^{\mathrm{i}}
\end{aligned}
$$

The integration constants are given by:

$$
\begin{aligned}
& a_{1}^{0}=\frac{n_{1}}{n} ; a_{2}^{0}=\frac{n_{2}}{n} ; a_{3}^{0}=\frac{n_{3}}{n} ; a_{4}^{0}=\frac{n_{4}}{n} \\
& a_{1}^{1}=\frac{n_{5}}{n} ; a_{2}^{1}=\frac{n_{6}}{n} ; a_{3}^{1}=\frac{n_{7}}{n} ; a_{4}^{1}=\frac{n_{8}}{n} \\
& a_{1}^{2}=\frac{n_{9}}{n} ; a_{2}^{2}=\frac{n_{10}}{n} ; a_{3}^{2}=\frac{n_{11}}{n} ; a_{4}^{2}=\frac{n_{12}}{n}
\end{aligned}
$$

\begin{tabular}{|c|c|c|c|c|c|c|c|c|c|c|c|}
\hline $\begin{array}{c}\mathrm{Ele}_{41}(0)+ \\
+\rho{ }^{2}{ }^{2} \mathrm{e}_{21}(0)\end{array}$ & $\begin{array}{c}\mathrm{EIe}_{42}(0)+ \\
+\rho \mathrm{I} \Omega^{2} \mathrm{e}_{22}(0)\end{array}$ & $\begin{array}{c}\mathrm{EIe}_{43}(0)+ \\
+\rho \mathrm{I} \Omega^{2} \mathrm{e}_{23}(0)\end{array}$ & $\begin{array}{c}\mathrm{Ele}_{44}(0)+ \\
+\rho \mathrm{I} \Omega^{2} \mathrm{e}_{24}(0)\end{array}$ & 0 & 0 & 0 & 0 & 0 & 0 & 0 & 0 \\
\hline$e_{31}(0)$ & $e_{32}(0)$ & $\mathrm{e}_{33}(0)$ & $\mathrm{e}_{34}(0)$ & 0 & 0 & 0 & 0 & 0 & 0 & 0 & 0 \\
\hline $\begin{array}{l}\mathrm{k}_{33} \mathrm{e}_{11}\left(\mathrm{l}_{1}\right)- \\
-\mathrm{EIe}_{41}\left(\mathrm{l}_{1}\right)\end{array}$ & $\begin{array}{l}\mathrm{k}_{33} \mathrm{e}_{12}\left(\mathrm{l}_{1}\right)- \\
-\mathrm{EIe}_{42}\left(\mathrm{l}_{1}\right)\end{array}$ & $\begin{array}{l}\mathrm{k}_{33} \mathrm{e}_{13}\left(\mathrm{l}_{1}\right)- \\
-\mathrm{EIe}_{43}\left(\mathrm{l}_{1}\right)\end{array}$ & $\begin{array}{l}\mathrm{k}_{33} \mathrm{e}_{14}\left(\mathrm{l}_{1}\right)- \\
-\mathrm{EIe}_{44}\left(\mathrm{l}_{1}\right)\end{array}$ & $\mathrm{EIe}_{41}\left(1_{1}\right)$ & $\mathrm{EIe}_{42}\left(\mathrm{l}_{1}\right)$ & $\mathrm{EIe}_{43}\left(\mathrm{l}_{1}\right)$ & $\mathrm{EIe}_{44}\left(1_{1}\right)$ & 0 & 0 & 0 & 0 \\
\hline $\begin{array}{l}\mathrm{k}_{54} \mathrm{e}_{21}\left(\mathrm{l}_{1}\right)- \\
-\mathrm{EIe}_{31}\left(\mathrm{l}_{1}\right)\end{array}$ & $\begin{array}{l}\mathrm{k}_{54} \mathrm{e}_{22}\left(\mathrm{l}_{1}\right)- \\
-\mathrm{EIe}_{32}\left(\mathrm{l}_{1}\right)\end{array}$ & $\begin{array}{l}\mathrm{k}_{54} \mathrm{e}_{23}\left(\mathrm{l}_{1}\right)- \\
-\mathrm{EIe}_{33}\left(\mathrm{l}_{1}\right)\end{array}$ & $\begin{array}{l}\mathrm{k}_{54} \mathrm{e}_{24}\left(\mathrm{l}_{1}\right)- \\
-\mathrm{EIe}_{34}\left(\mathrm{l}_{1}\right)\end{array}$ & $\mathrm{EIe}_{31}\left(\mathrm{l}_{1}\right)$ & $\mathrm{EIe}_{32}\left(\mathrm{l}_{1}\right)$ & $\mathrm{EIe}_{33}\left(\mathrm{l}_{1}\right)$ & $\mathrm{EIe}_{34}\left(\mathrm{l}_{1}\right)$ & 0 & 0 & 0 & 0 \\
\hline$-e_{11}\left(1_{1}\right)$ & $-e_{12}\left(1_{1}\right)$ & $-\mathrm{e}_{13}\left(\mathrm{l}_{1}\right)$ & $-e_{14}\left(1_{1}\right)$ & $e_{11}\left(l_{1}\right)$ & $\mathrm{e}_{12}\left(\mathrm{l}_{1}\right)$ & $\mathrm{e}_{13}\left(\mathrm{l}_{1}\right)$ & $e_{14}\left(l_{1}\right)$ & 0 & 0 & 0 & 0 \\
\hline$-e_{21}\left(1_{1}\right)$ & $-e_{22}\left(1_{1}\right)$ & $-e_{23}\left(1_{1}\right)$ & $-e_{24}\left(1_{1}\right)$ & $\mathrm{e}_{21}\left(\mathrm{l}_{1}\right)$ & $e_{22}\left(l_{1}\right)$ & $\mathrm{e}_{23}\left(\mathrm{l}_{1}\right)$ & $\mathrm{e}_{24}\left(\mathrm{l}_{1}\right)$ & 0 & 0 & 0 & 0 \\
\hline 0 & 0 & 0 & 0 & $\begin{array}{l}\mathrm{k}_{33} \mathrm{e}_{11}\left(\mathrm{l}_{2}\right)- \\
-\mathrm{EIe}_{41}\left(\mathrm{l}_{2}\right)\end{array}$ & $\begin{array}{l}\mathrm{k}_{33} \mathrm{e}_{12}\left(\mathrm{l}_{2}\right)- \\
-\mathrm{EIe}_{42}\left(\mathrm{l}_{2}\right)\end{array}$ & $\begin{array}{l}\mathrm{k}_{33} \mathrm{e}_{13}\left(\mathrm{l}_{2}\right)- \\
-\mathrm{EIe}_{43}\left(\mathrm{l}_{2}\right)\end{array}$ & $\begin{array}{l}\mathrm{k}_{33} \mathrm{e}_{14}\left(\mathrm{l}_{2}\right)- \\
-\mathrm{EIe}_{44}\left(\mathrm{l}_{2}\right)\end{array}$ & $\mathrm{EIe}_{41}\left(1_{2}\right)$ & $\mathrm{EIe}_{42}\left(\mathrm{l}_{2}\right)$ & $\mathrm{EIe}_{43}\left(1_{2}\right)$ & $\mathrm{EIe}_{44}\left(\mathrm{l}_{2}\right)$ \\
\hline 0 & 0 & 0 & 0 & $\begin{array}{l}\mathrm{k}_{54} \mathrm{e}_{21}\left(\mathrm{l}_{2}\right)- \\
-\mathrm{EIe}_{31}\left(\mathrm{l}_{2}\right)\end{array}$ & $\begin{array}{l}\mathrm{k}_{54} \mathrm{e}_{22}\left(\mathrm{l}_{2}\right)- \\
-\mathrm{EIe}_{32}\left(\mathrm{l}_{2}\right)\end{array}$ & $\begin{array}{l}\mathrm{k}_{54} \mathrm{e}_{23}\left(\mathrm{l}_{2}\right)- \\
-\mathrm{EIe}_{33}\left(\mathrm{l}_{2}\right)\end{array}$ & $\begin{array}{l}\mathrm{k}_{54} \mathrm{e}_{24}\left(\mathrm{l}_{2}\right)- \\
-\mathrm{EIe}_{34}\left(\mathrm{l}_{2}\right)\end{array}$ & $\mathrm{EIe}_{31}\left(\mathrm{l}_{2}\right)$ & $\mathrm{EIe}_{32}\left(\mathrm{l}_{2}\right)$ & $\mathrm{EIe}_{33}\left(\mathrm{l}_{2}\right)$ & $\mathrm{EIe}_{34}\left(\mathrm{l}_{2}\right)$ \\
\hline 0 & 0 & 0 & 0 & $-e_{11}\left(l_{2}\right)$ & $-e_{12}\left(l_{2}\right)$ & $-\mathrm{e}_{13}\left(\mathrm{l}_{2}\right)$ & $-e_{14}\left(l_{2}\right)$ & $\mathrm{e}_{11}\left(\mathrm{l}_{2}\right)$ & $\mathrm{e}_{12}\left(\mathrm{l}_{2}\right)$ & $\mathrm{e}_{13}\left(\mathrm{l}_{2}\right)$ & $\mathrm{e}_{14}\left(\mathrm{l}_{2}\right)$ \\
\hline 0 & 0 & 0 & 0 & $-e_{21}\left(1_{2}\right)$ & $-e_{22}\left(l_{1}\right)$ & $-e_{23}\left(1_{2}\right)$ & $-e_{24}\left(1_{2}\right)$ & $\mathrm{e}_{21}\left(\mathrm{l}_{2}\right)$ & $\mathrm{e}_{22}\left(1_{2}\right)$ & $\mathrm{e}_{23}\left(1_{2}\right)$ & $\mathrm{e}_{24}\left(1_{2}\right)$ \\
\hline 0 & 0 & 0 & 0 & 0 & $\rho \mathrm{I} \Omega^{2} \mathrm{e}_{21}(1)$ & 0 & 0 & $\begin{array}{c}\mathrm{EIe}_{41}(\mathrm{l})+ \\
+\mathrm{m}_{\mathrm{p}} \Omega^{2} \mathrm{e}_{11}(\mathrm{l})\end{array}$ & $\begin{array}{c}\mathrm{Ele}_{42^{(1)+}} \\
+\rho \Omega^{2} \mathrm{e}_{22}(\mathrm{l}) \\
+\mathrm{m}_{\mathrm{p}} \Omega^{2} \mathrm{e}_{12}(\mathrm{l})\end{array}$ & $\begin{aligned} & \mathrm{EIe}_{43^{(1)+}} \\
+ & \Omega^{2}{ }^{2} \mathrm{e}_{23}(\mathrm{l}) \\
+ & \mathrm{m}_{\mathrm{p}} \Omega^{2} \mathrm{e}_{13}(\mathrm{l})\end{aligned}$ & $\begin{array}{c}\mathrm{Ele}_{44^{(1)+}} \\
+\rho \Omega^{2} \mathrm{e}_{24}(\mathrm{l}) \\
+\mathrm{m}_{\mathrm{p}} \Omega^{2} \mathrm{e}_{14}{ }^{(1)}\end{array}$ \\
\hline 0 & 0 & 0 & 0 & 0 & 0 & 0 & 0 & $\begin{array}{c}\mathrm{EIe}_{31}(\mathrm{l})- \\
-\mathrm{J} \Omega^{2} \mathrm{e}_{11}(\mathrm{l})\end{array}$ & $\begin{array}{c}\mathrm{EIe}_{32}(1)- \\
-\mathrm{J} \Omega^{2}{ }^{2} \mathrm{e}_{12}(1)\end{array}$ & $\begin{array}{c}\mathrm{EIe}_{33}(1)- \\
-\mathrm{J} \Omega^{2}{ }^{2}{ }_{13}(1)\end{array}$ & $\begin{array}{c}\mathrm{EIe}_{34}(1)- \\
-\mathrm{J} \Omega^{2} \mathrm{e}_{14}{ }^{(1)}\end{array}$ \\
\hline
\end{tabular}

In this case, we can study the system stability by analyzing the expression at the denominator of integration constants $\mathrm{a}_{1}^{\mathrm{i}}, \mathrm{a}_{2}^{\mathrm{i}}, \mathrm{a}_{3}^{\mathrm{i}}, \mathrm{a}_{4}^{\mathrm{i}}, \mathrm{i} \in\{0,1,2\}$. The determinant $\mathrm{n}$ is given by: 
Computing this determinant gives an expression of the form [8]:

$$
\begin{aligned}
& \mathrm{P}(\mathrm{s}) \quad=\left(0.206 \cdot \Omega^{4}+264.9 \cdot \Omega^{2}+16.0214 \cdot 10^{-6}\right) \cdot \mathrm{s}^{6}+\left(-35.11 \cdot \Omega^{6}-3672 \cdot 10^{9} \cdot \Omega^{4}+41.08 \cdot 10^{28} \cdot \Omega^{2}+\right. \\
& \quad+2534.609) \cdot \mathrm{s}^{4}+6.98 \cdot 10^{-6} \cdot \Omega \cdot \mathrm{s}^{2}+\left(-37.82 \cdot 10^{-14} \cdot \Omega^{8}+2.39 \cdot 10^{9} \cdot \Omega^{6}+245.27 \cdot 10^{-7} \cdot \Omega^{5}+3.35\right. \\
& \left.\quad+10^{15} \cdot \Omega^{4}-57.61 \cdot \Omega^{3}+456 \cdot 10^{15} \cdot \Omega^{2}+182.6 \cdot 10^{15} \cdot \Omega+1.08 \cdot 10^{16}\right) \cdot \mathrm{s}^{2}+107.24 \cdot 10^{-5} \cdot \Omega \mathrm{s}+ \\
& \quad+\left(6.86 \cdot 10^{-6} \cdot \Omega^{8}+3.19 \cdot 10^{13} \cdot \Omega^{6}+8.3 \cdot 10^{8} \cdot \Omega^{5}+37.82 \cdot 10^{-14} \cdot \Omega^{2}+1.27 \cdot 10^{22} \cdot \Omega^{4}+\right. \\
& \left.\quad+1.76 \cdot 10^{24} \cdot \Omega^{2}-4.57 \cdot 10^{14}\right)
\end{aligned}
$$

Where:

- computations were made under the assumptions $\sin \alpha \approx \alpha, \cos \alpha \approx 1$ for small domains of $\Omega$;

- $\Omega$ represents the angular rotation speed of the spindle.

Using special MATLAB programs, there were established the roots of the polynomial $\mathrm{P}(\mathrm{s})=0$ [3]. These have the form:

$\mathrm{s}_{1,2}= \pm \mathrm{j} \omega_{1}$, which represents a continuous sinusoidal component;

$\mathrm{s}_{3,4}=\sigma_{2} \pm \mathrm{j} \omega_{2}$, which represents a (stabile) damping component;

$\mathrm{s}_{5,6}=\sigma_{3} \pm \mathrm{j} \omega_{2}$, a component which amplifies the instability character of the movement.

The solutions obtained for $\mathrm{P}(\mathrm{s})=0$ are:

$$
\begin{aligned}
& s_{1,2}= \pm 7.0596 \cdot 10^{11} j \\
& s_{3,4}=-42 \cdot 10^{-2} \pm 42 \cdot 10^{-2} j \\
& s_{5,6}=42 \cdot 10^{-2} \pm 42 \cdot 10^{-2} j
\end{aligned}
$$

Based on Mihailov's stability criterion (Fig. 1), given the maximal degree of $\mathrm{P}(\mathrm{s})$, in order for the system to be stabile $\mathrm{P}(\mathrm{j} \omega)$ must cover all $\mathrm{u}=6$ planes, corresponding to the highest power exponent.

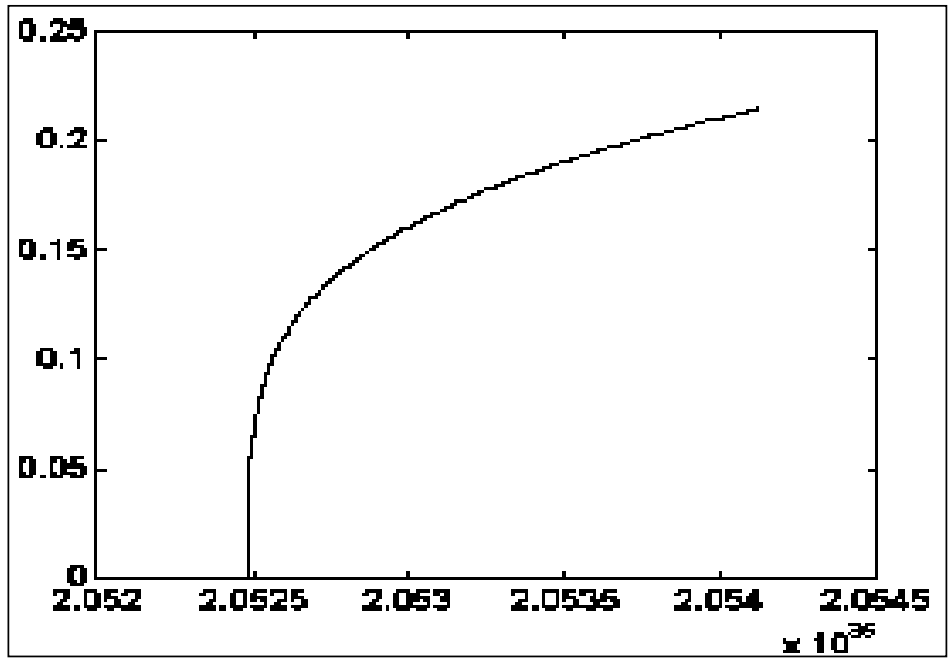

Figure 1

In our case, it can be observed that quadrant (1) is only partially covered, which outlines the instability character of the internal grinding process in this direction, a fact that was shown also in specialized literature. 
The solutions of the vibration movement can be written under the general form [9]:

$\mathrm{v}_{3}\left(\mathrm{t}, \mathrm{x}_{1}\right)=\mathrm{A}(\mathrm{t})+\mathrm{A}^{\prime}(\mathrm{t}) \cdot \mathrm{x}_{1}$

where: $\quad A(t)=a_{1} \sin \left(\omega_{1} t+\phi_{1}\right)+a_{2} e^{-\sigma_{2} t} \sin \left(\omega_{2} t+\phi_{2}\right)+a_{3} e^{\sigma_{3} t} \sin \left(\omega_{3} t+\phi_{3}\right)$

and $\quad \mathrm{A}^{\prime}(\mathrm{t})=\mathrm{a}_{1}^{\prime} \sin \left(\omega_{1} \mathrm{t}+\phi_{1}^{\prime}\right)+\mathrm{a}_{2}^{\prime} \mathrm{e}^{-\sigma_{2} \mathrm{t}} \sin \left(\omega_{2} \mathrm{t}+\phi_{2}^{\prime}\right)+\mathrm{a}_{3}^{\prime} \mathrm{e}^{\sigma_{3} \mathrm{t}} \sin \left(\omega_{3} \mathrm{t}+\phi^{\prime}{ }_{3}\right)$

with $\quad \sigma_{2}=\sigma_{3}$ and $\omega_{2}=\omega_{3}$

Based on these results, it was realized a simulation for the three components of the vibration movements denoted by $\mathrm{C}_{1}(\mathrm{t}), \mathrm{C}_{2}(\mathrm{t})$ and $\mathrm{C}_{3}(\mathrm{t})$, in which:

- according to Fig. 2 (a, b, c), we have the real case:

$$
\begin{aligned}
& \omega_{1}=7 \cdot 10^{11} \mathrm{sec}^{-1} \\
& \omega_{2}=\omega_{3}=0.42 \mathrm{sec}^{-1} \\
& \sigma_{2}=\sigma_{3}=0.42
\end{aligned}
$$

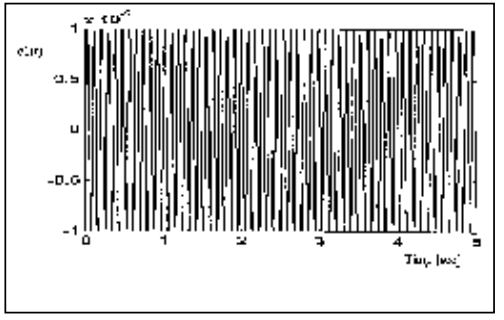

Figure 2a

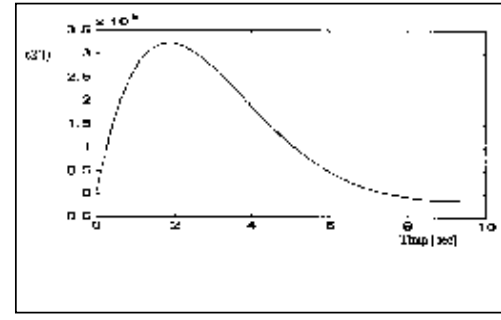

Figure 2b

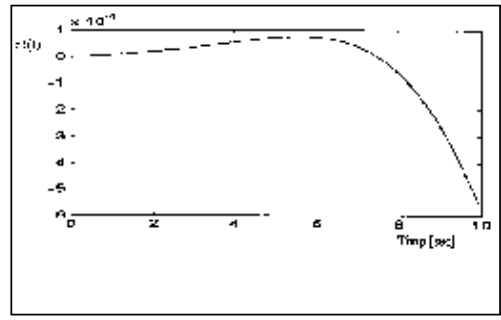

Figure 2c

$$
\begin{aligned}
& \omega_{1}=7 \mathrm{sec}^{-1} \\
& \omega_{2}=\omega_{3}=4.2 \mathrm{sec} \\
& \sigma_{2}=\sigma_{3}=0.42
\end{aligned}
$$

- according to Fig. 3 (a, b, c), we have the real case: $\quad \omega_{2}=\omega_{3}=4.2 \mathrm{sec}^{-1}$

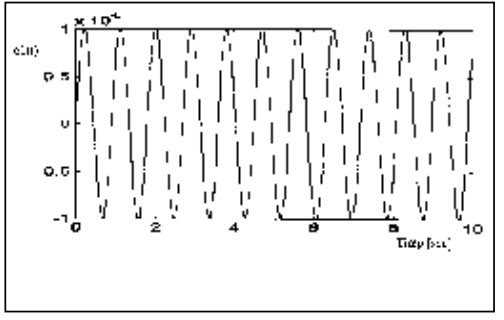

Figure 3a

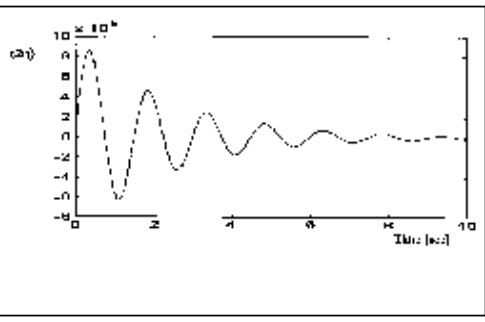

Figure 3b

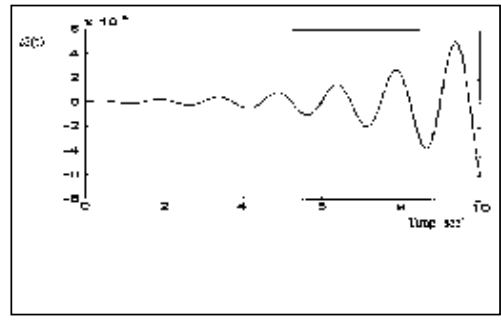

Figure 3c

$$
\begin{array}{ll} 
& \omega_{1}=70 \mathrm{sec}^{-1} \\
\text { - according to Fig. } 4 \text { (a, b, c), we have the real case: } & \omega_{2}=\omega_{3}=42 \mathrm{sec}^{-1}
\end{array}
$$

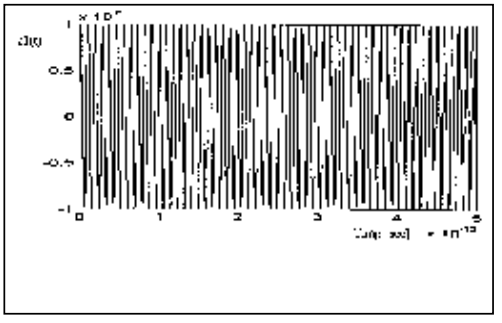

Figure 4a

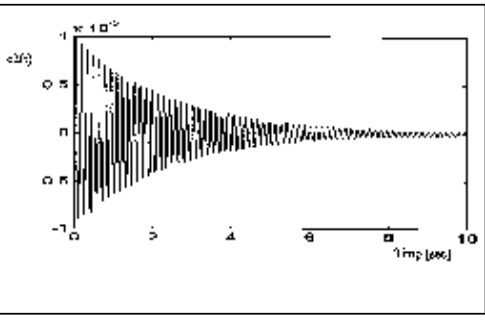

Figure 4b

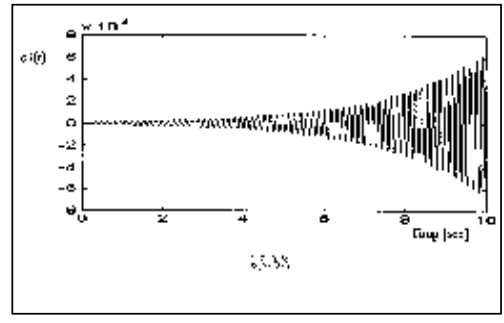

Figure 4c 
The analysis of the main spindle vibrations in transversal direction at internal grinding shows the specific characteristic of this grinding process, that is the dynamic instability which determines an increase of the amplitude level in a short period, due to the loading of the abrasive tool, which leads to a deterioration in the surface quality and consequently to processing stop.

\section{CONCLUSIONS}

1) Stability analysis of the spindle in transversal direction is performed based on the $1^{\text {st }}$ degree equation system that describe the transversal vibration movement.

2) Using Mihailov's stability criterion emphasizes the partial instability characteristic of the process in this direction, a fact also emphasized in other works from specialty literature.

3) Finally, there is presented a simulation of the three components of the vibration movement $C_{1}(t), C_{2}(t)$, $\mathrm{C}_{3}(\mathrm{t})$ for representative values of the frequency.

\section{SOURCES OF FUNDING}

None.

\section{CONFLICT OF INTEREST}

None.

\section{ACKNOWLEDGMENT}

None.

\section{REFERENCES}

[1] Abele, E., Altintas, Y. \& Brecher, C., 2010. Machine tool spindles. CIRP Annals Manufacturing Technology, 59(781-802).

[2] He, X., 2015. Recent development in reliability analysis of NC machine tools. Int J Adv Manuf Technol.

[3] Jorgensen, B. \& Shin, Y., 1996. Dynamics of Machine Tool Spindle/Bearing Systems Under Thermal Growth, Journal of tribology. 119(4), 875-882 (Oct 01, 1997), 119(4), p. 8.

[4] Mobley, R. K., 2000. Vibration Fundamentas. s.l.:Butterworth-Heinemann.

[5] Weck, M. \& Koch, A., 1993. Spindle-Bearing systems for high speed aplications in machine tools.

[6] Quintana, G., de Ciurana, J. \& Campa, F., 2009. Chapter 3: Machine tool Spindles. In: Machine tools for High Performance Machining.López de Lacalle, L. N.; Lamikiz, A. s.l.:Springer.

[7] Popescu, D., Bolcu, D., Rizescu, S. - Establishment of the movement equations which define the vibrations of the spindle at internal grinding, The 10th DAAAM International Symposium, Vienna, Austria, 21-23 October 1999, pp. 445-447.

[8] Popescu, D. - Theoretical and experimental contributions regarding improving the processing precision at internal grinding machines, PhD Thesis, Univ. P.R., Bucharest, 1999, pp. 87-90.

[9] Popescu, D., Ghionea, A. - Analysis of the vibration of the main spindle in transversal direction at internal grinding, Acta Univesitas CIBINIENSIS Vol. XXXVII, pp. 147-151. 